\title{
Long-Term Maintenance of Presynaptic Function in the Absence of Target Muscle Fibers
}

\author{
Anna Dunaevsky and Elizabeth A. Connor \\ Department of Biology, Neuroscience and Behavior Graduate Program, University of Massachusetts, Amherst, \\ Massachusetts 01003
}

\begin{abstract}
Here we have investigated the role of the muscle fiber in the maintenance of presynaptic function at frog motor nerve terminals. Muscle fibers were selectively damaged and prevented from regenerating while leaving the motor innervation intact. Presynaptic activity of the resulting target-deprived nerve terminals was assayed using the fluorescent dye, FM1-43. FM1-43 stains nerve terminals in an activity-dependent fashion in that it incorporates into synaptic vesicles and can be released upon stimulation. We demonstrate that nerve terminals permanently deprived of target for 1 to 5 months maintain the ability to release and recycle synaptic vesicles in response to stimulation. The level of presynaptic function of target-deprived nerve terminals was not detectably different from that of nerve terminals in contact with muscle fibers. The remarkable maintenance of presynaptic function in these target-deprived nerve terminals indicates that the molecular machinery required for vesicular release is maintained in a functional state for long periods without target muscle fibers.
\end{abstract}

[Key words: neuromuscular junction, peanut agglutinin (PNA), FM1-43, synaptic basal lamina, nerve terminal, synapse maintenance]

Once formed, mature motor nerve terminals are remarkably stable structures (for review see Wernig and Herrera, 1986; Lichtman et al., 1987; Herrera et al., 1990). At the neuromuscular junction, presynaptic specializations of the motor nerve terminal are precisely aligned with components of the muscle fiber postsynaptic apparatus. For example, presynaptic release sites, called active zones, directly appose muscle membrane infoldings and concentrations of acetylcholine receptors (AChR) (Kuffler and Yoshikami, 1975). Organized at active zones are synaptic vesicles and the molecules responsible for neurotransmitter release (Robitäille et al., 1990; Cohen et al., 1991; Bennett et al., 1992; Ushkaryov et al., 1992; Robitaille et al., 1993). The stability of effective synaptic transmission at mature neuromuscular junctions requires the coordinated maintenance of the presynaptic and postsynaptic structures at the molecular level. While the

\footnotetext{
Received Apr. 25, 1995; accepted May 4, 1995.

We thank V. Budnik, R. Murphey, M. Smith, and Y. M. Yao for helpful advice in preparing this manuscript, $P$. Wadsworth for use of photographic equipment and comments on the manuscript, and W. Johnston for her technical assistance. We also thank Amherst College and Mount Holyoke College for allowing us to use their $\mathrm{x}$-irradiation facilities. This work was supported by NIH NS26879.

Correspondence should be addressed to Elizabeth A. Connor at the above address.

Copyright 1995 Society for Neuroscience $0270-6474 / 95 / 156137-(08 \$ 05.00 / 0$
}

identification of proteins such as agrin (Bowe and Fallon, 1995) and ARIA (Falls et al., 1990, 1993) has provided clues as to the formation and maintenance of postsynaptic specializations, only recently have the mechanisms of the development and maintenance of nerve terminal organization been addressed (BaliceGordon and Lichtman, 1994; Noakes et al., 1995).

One possible mechanism for the maintenance of nerve terminals is that retrograde signals from target muscle fibers provide stability to nerve terminals (reviewed in Connor and Smith, 1994). In this study, we sought to determine the role of the muscle fiber in the maintenance of nerve terminal activity by examining presynaptic function in nerve terminals deprived of target for long periods of time. Previous morphological studies revealed that most frog nerve terminals persist at synaptic sites for long periods after muscle removal (Yao, 1988b), whereas in mammals, immediate retraction of nerve terminal branches resulted from target deprivation (Rich and Lichtman, 1989). Here, we used living frog nerve terminals to assay presynaptic activity after several months of target deprivation.

Since the absence of muscle fibers makes conventional electrophysiological determination of transmitter release impossible, we assayed presynaptic activity in target-deprived nerve terminals using the styryl dye, FM1-43. There is strong evidence that FM143 is incorporated into synaptic vesicles upon stimulation and can be used as an optical assay of presynaptic function. Previous experiments have demonstrated that FM1 43 staining and destaining is calcium dependent, its distribution is consistent with that of synaptic vesicles, synapsin, and synaptotagmin, and mutation of the endocytotic pathway blocks FM1-43 staining (Betz et al., 1992; Ryan et al., 1993; Ramaswami et al., 1994). Here we report that frog nerve terminals permanently deprived of muscle fibers not only remain at synaptic sites after target removal but also retain their ability to release and recycle synaptic vesicles for up to 5 months. Further, the level of presynaptic function of targetdeprived nerve terminals was not detectably different from that of nerve terminals in contact with muscle fibers. These data indicate that the molecular machinery required for release and recycling of synaptic vesicles is maintained for long periods without muscle, suggesting that the signals required for the continued synthesis and proper localization of these presynaptic molecules remain intact in the absence of a target.

\section{Materials and Methods}

Surgery. Adult male frogs, Rana pipiens, were anesthetized by immersion in $0.2 \%$ tricaine methane sulfonate $(\mathrm{pH} \mathrm{7.2)}$ and cooled on ice. Cutaneous pectoris muscles, situated just beneath the skin of the thorax, were exposed, and muscle fibers were damaged while leaving the motor innervation intact (Yao, 1988b; Anglister, 1991). Specifically, careful incisions were made across myofibers and between nerve arbors. Sub- 
sequently, junctional sites were separated from nonjunctional portions of fibers that were discarded. Within 2 weeks, the remaining segments of myofibers degenerated and were phagocytized, leaving empty basal lamina sheaths of muscle fibers with original nerve terminals intact. These preparations are referred to as innervated sheaths. Regeneration of myofibers was prevented by $\mathrm{x}$-irradiation of the thorax on 3 consecutive days at the time of surgery (total dose/d - 3900 rads, Sames el al., 1978; Yao, 1988b). Occasionally innervated sheaths had persisting or regenerated muscle fibers; large-diameter fibers persisted in sheaths due to insufficient surgical damage, while small diameter fibers were likely to have regenerated. Surviving muscle fibers were identified by the presence of striations in bright-field microscopy, allowing us to distinguish target-deprived nerve terminals from terminals in contact with muscle fibers. We limited our observations of target-deprived terminals to regions of sheaths that contained at most a few fibers and did not analyze synaptic sites in fiber-rich regions. Further, we discarded preparations with a high fiber density ( $>60$ muscle fibers/sheath) since these usually had few fiber-free areas.

Staining. One to 5 months after muscle damage, innervated sheaths were dissected in normal frog Ringer's solution $(160 \mathrm{mM} \mathrm{NaCl}, 4 \mathrm{~mm}$ $\mathrm{KCl}, 1.8 \mathrm{mM} \mathrm{CaCl}_{2}, 1 \mathrm{~mm} \mathrm{NaH} \mathrm{PO}_{4}, \mathrm{pH} 7.2$ ). Synaptic sites were identified by staining with rhodamine-conjugated peanut agglutinin (PNA, Sigma, St. Louis, MO, $33 \mu \mathrm{g} / \mathrm{ml}$ in Ringer's solution for $15 \mathrm{~min}$, Ko, 1987). Some preparations were stained with rhodamine-conjugated $\alpha$-bungarotoxin (Molecular Probes, Eugene, OR; $2 \mu \mathrm{g} / \mathrm{ml}, 15 \mathrm{~min}$ ) to mark AChRs and viewed prior to staining with PNA.

As a functional assay for the release and recycling of synaptic vesicles, innervated sheaths were stained with the fluorescent dye, FM 1-43, (Molecular Probes, Eugene, OR; 5 min in $2 \mu \mathrm{M}$ FM1-43 in high $\mathrm{K}^{+}$ Ringer's solution: $60 \mathrm{~mm} \mathrm{~K}^{+}$with reduced external $\left.\left[\mathrm{Na}^{+}\right]\right)$, and washed in normal Ringer's solution. Nerve stimulation was produced by depolarization with a high $\mathrm{K}^{+}$solution to ensure that each nerve terminal was activated. Consistent with previous observations, we found a similar pattern of stain with evoked nerve stimulation $(10 \mathrm{~Hz}, 10 \mathrm{~min}$, Betz et al., 1992; Ramaswami et al., 1994). A region of an innervated sheath with no or few muscle fibers was selected, and the locations of individual synaptic sites were identified by PNA stain. A synaptic site was found to be either on or off a muscle fiber by bright-field microscopy and was scored as stained or unstained by FM1-43. We then tested whether FM 1-43-stained nerve terminals could be depleted of stain by either direct electrical stimulation of the cutaneous pectoris nerve $(1 \mathrm{~Hz}$ continuous stimulation for $10 \mathrm{~min}$, with $5 \mathrm{sec} 10 \mathrm{~Hz}$ trains every 30 $\mathrm{sec}$ ) or $10 \mathrm{~min}$ in high $\mathrm{K}^{+}$Ringer's solution (Betz et al., 1992).

Analysis of fluorescence intensity. Synaptic sites that were clearly visualized and well stained by PNA were selected. Nerve terminals were visualized with a Dage VE-1000 SIT camera, at constant settings of black level, gain, and voltage. Images were digitized, averaged $(\times 16)$, and stored using the NIH Image software package version 1.54. The intensity of the FM1-43 stain was determined from an inverted image by calculating the summed gray value for an outlined nerve terminal area from which a background value had been subtracted. A background gray value was determined for a nonsynaptic region of each image. FM1-43 staining intensity of a nerve terminal was expressed as the summed gray value/ micrometer length of nerve terminal. Some error in the quantification of intensity may have been introduced by the mercury arc lamp. Although some variation in the light source likely occurred, we believe that it did not alter the outcome of these experiments, since images from different experimental groups were collected over 2 months in random order. Further, the distribution of FMl-43 staining intensities from individual preparations in the same experimental group did not vary over the 2 month collection period. Some activity-independent stain, associated with nuclei of terminal Schwann cells (Betz et al., 1992), was generally not included in the quantitation measurement.

Light microscopy. To assess the integrity of axons, innervated sheath preparations were fixed, stained for cholinesterase and myelin following fluorescence microscopy, and examined in whole mount by light microscopy (Connor et al., 1994).

Immunohistochemistry. Innervated sheaths were frozen, sectioned (10 $\mu \mathrm{m}$ sections), and stained for immunohistochemistry (Connor et al., 1994). Basal laminae were identified with a rabbit polyclonal antibody against laminin (gift of R. Timpl) and FITC-conjugated goat anti-rabbit IgG (Cappel Research Products, Durham, NC). Muscle fibers were stained with rhodamine-conjugated phalloidin (Molecular Probes, Eugene, OR).

\section{Results}

To address the role of the muscle fiber in the functional stability of motor nerve terminals, muscle fibers of cutaneous pectoris muscles were selectively damaged and prevented from regenerating, while the motor innervation remained intact. This resulted in muscle fiber degeneration, leaving empty sheaths of muscle fiber basal lamina (Sanes et al., 1978; Yao, 1988b; Anglister, 1991). Although muscle fibers were totally absent from some innervated sheaths $(n=6)$, some fibers remained in others $[$ mean $=18 \pm 18 \mathrm{SD}$ muscle fibers per innervated sheath $(n=$ 34 ); note that in innervated muscle mean $=480 \pm 45$ muscle fibers, Connor and McMahan, 19871. To confirm that all synaptic sites in contact with surviving muscle fibers could be identified, the muscle fibers in several innervated sheaths were counted using bright-field microscopy and their relative positions were noted. These sheaths were then frozen sectioned and stained to visualize basal laminae and muscle fibers (Tig. $1 A, B$ ). When we compared the number and relative positions of myofibers in sections to that determined in whole mount, we found that all muscle fibers observed in frozen sections had been previously identified by the presence of striations. Thus, muscle fibers in innervated sheaths were identified by bright-field microscopy with great accuracy, making the misidentification of target-deprived nerve terminals unlikely. Examination of whole-mount preparations stained for cholinesterase and myelin revealed that the density of synaptic sites and the innervation pattern in innervated sheaths was unaffected by muscle removal and similar to that of normal muscle (Fig. $1 C, D$ ). The presynaptic function of target-deprived nerve terminals in innervated sheaths was compared to that of normal nerve terminals using the styryl dye, FM1-43 (Betz et al., 1992).

\section{Presynaptic function in normal muscles}

Motor nerve terminals in normal muscle were stained with FM143 in an activity-dependent manner (Betz et al., 1992). Following nerve stimulation with high $\mathrm{K}^{+}$in the presence of dye, beadlike fluorescence appeared in nerve terminals (Fig. 2B). This linear array of FM1-43 stain, representing populations of stained synaptic vesicles clustered at active zones along the nerve terminal (Betz et al., 1992), occupied the space delineated by the PNA stain that marks the basal laminae of the terminal Schwann cell and the synapse (Fig. $2 A, \mathrm{Ko}, 1987$ ). I abeled nerve terminals were subsequently destained by stimulation in the absence of dye (Fig. 2C). Other structures, such as myelin and Schwann cell vacuoles, were stained with FM1-43 but not destained (Betz et al., 1992), and served as orienting landmarks.

\section{Presynaptic function $1-5$ months after muscle damage}

In innervated sheath preparations deprived of muscle fihers for 1 month ( $n=17,10$ frogs), synaptic sites were clearly identified by PNA stain. At the majority of target-deprived synaptic sites, the pattern of PNA stain was not different from that in normal muscles (Figs. 2A, 3A). Following stimulation in the presence of dye, nerve terminals at target-deprived synaptic sites were stained by FM1-43 in a manner identical to that observed in nerve terminals of normal muscle (Figs. $2 B, 3 B$ ); FM1-43 stain occupied the space delineated by PNA stain. Further, these nerve terminals were depleted of stain when stimulated in the absence of dye (Fig. 3C).

To confirm that nerve terminals stained by FM1-43 were target deprived and not in contact with surviving muscle fibers, some preparations were stained with rhodamine-conjugated 

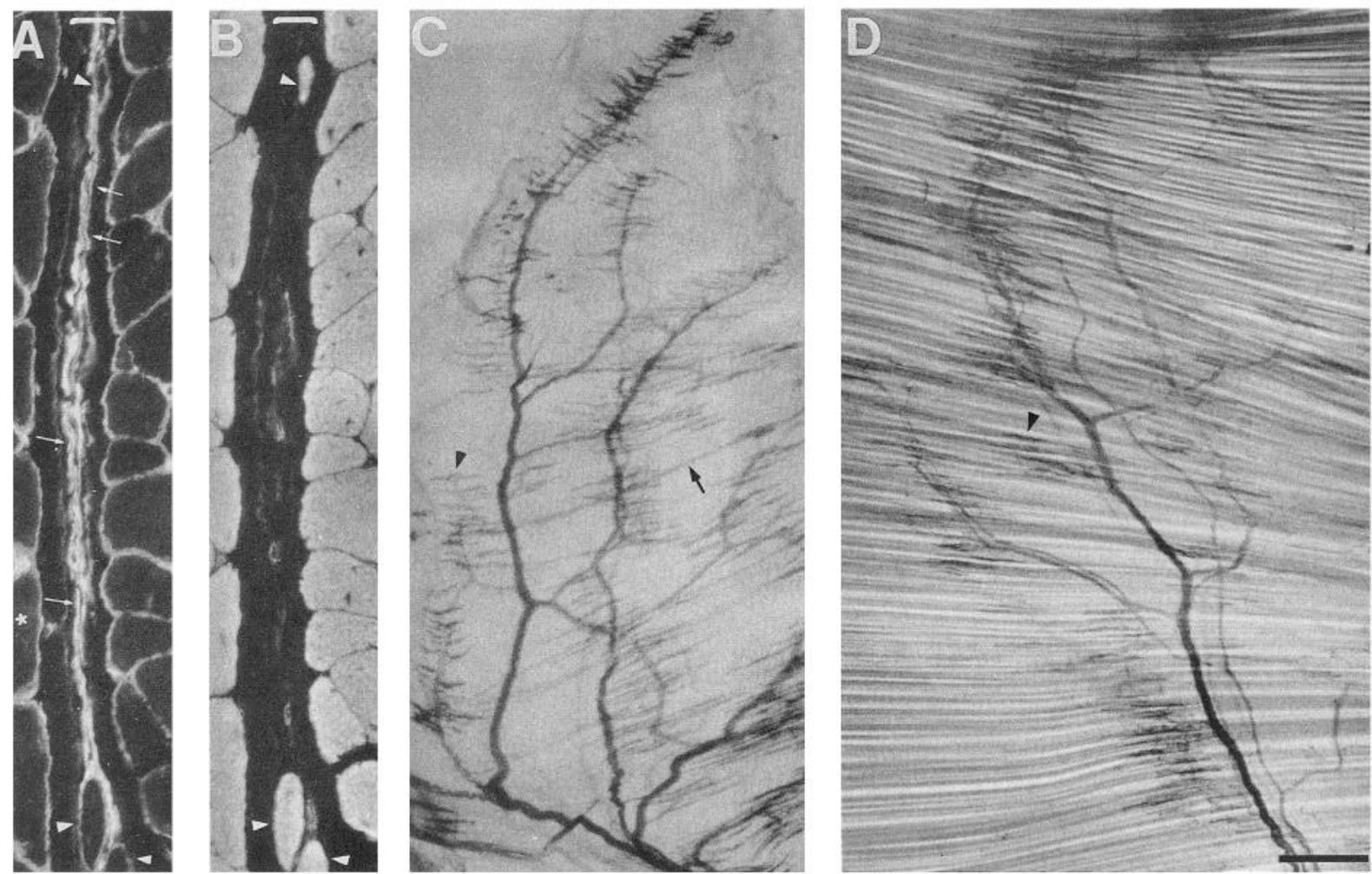

Figure 1. A and B, A frozen section of a 1 month innervated sheath (bracket) sandwiched between muscles. A, Most basal lamina sheaths, marked with a laminin antibody, are empty and collapsed (arrows), while a few contain persisting myofibers (arrowheads). B, Surviving myofibers are stained with phalloidin (arrowheads). $C$ and $D$. The density of synaptic sites and innervation pattern after muscle damage is similar to that in normal muscle. Whole-mount preparations of $(C)$ a 1 month innervated sheath and $(D)$ a normal muscle. Synaptic sites (arrowheads) are identified by cholinesterase stain. Myelin stain (osmium) marks the nerve and its branches. One of the remaining muscle fibers in the sheath $(C)$ is marked with an arrow. Bar, $(A$ and $B) 35 \mu \mathrm{m} ;(C) 590 \mu \mathrm{m} ;(D) 400 \mu \mathrm{m}$.

$\alpha$-bungarotoxin to mark AChRs. AChRs are concentrated at postsynaptic sites of regenerating as well as adult muscle fibers (Kuffler and Yoshikami, 1975; McMahan and Slater, 1984). No $\alpha$-bungarotoxin stain was associated with FM1-43-stained nerve terminals identified as target deprived by bright-field microscopy (Fig. 4). At synaptic sites on muscle fibers, however, FM1-43stained nerve terminals apposed narrow bands of stain marking AChRs in the muscle membrane.

Similar FM1-43 stain was observed in innervated sheaths whose muscle fibers were removed for longer time periods. We examined presynaptic function at target-deprived synaptic sites in 23 innervated sheaths either 2, 3, 4, or 5 months after muscle damage. At each time point, nerve terminals at PNA-stained synaptic sites were stained by FM1-43 (Fig. 5) and destained upon further stimulation (data not shown). Thus, nerve terminals deprived of target remain at synaptic sites and retain the ability to recycle and release synaptic vesicles for up to 5 months.

While many target-deprived synaptic sites were occupied by functional nerve terminals, as measured by FM1-43 staining, some were unstained by FM1-43 (Fig. 6A,B). Compared to control values, approximately $20 \%$ of target-deprived sites were unlabeled by FM1-43 1 and 5 months after muscle fiber removal (Table 1). Failure of a synaptic site to stain with FM1-43 may be a consequence of target loss; a nerve terminal may cease to function or may retract from the synaptic site. Alternatively, unstained synaptic sites may result from surgical damage. We believe that the failure of synaptic sites to stain with FM1-43 can be attributed to nerve damage during surgery for several reasons. The absence of FM1-43 stain was often correlated with synaptic sites only faintly stained by PNA (Fig. 6A,B). Since PNA stain is gradually lost from synaptic sites after denervation (Somasekhar and Ko, 1991), faint PNA staining is consistent with nerve terminal degeneration, although nerve terminal retraction may yield a similar result. Synaptic sites unstained by FM1-43 were often concentrated in an area of a sheath that showed symptoms of nerve degeneration when examined in whole mount. Also, there was no progressive increase in the proportion of synaptic sites unstained by FM1-43 as a function of time without target, as would be expected from nerve terminal retraction (Table 1). Thus, nerve damage resulting from surgery can reasonably account for the lack of FM1-43 staining at some synaptic sites, although we have not eliminated nerve terminal retraction due to loss of target as a possible cause (see Discussion).

At some synaptic sites, both on and off muscle fibers, the synaptic area delineated by PNA stain was not completely stained by FM1-43 (Figs. 5, 6). FM1-43 stain was not observed independently of PNA stain. The mismatch between PNA and FM1-43 stains resembles the ongoing remodeling observed at normal frog neuromuscular junctions (Herrera et al., 1990; Herrera and Werle, 1990; Chen et al., 1991; Chen and Ko, 1994). Although the net result of remodeling at target-deprived sites, reduction or expansion of a functional nerve terminal, was not addressed in this study, further investigation would be interesting since muscle fiber growth has been thought to be an important stimulus for synapse remodeling (Herrera and Werle, 1990).

An interesting feature of some target-deprived synaptic sites was a striking alteration in the extent of PNA stain. At these sites, PNA stain extended longitudinally well beyond the syn- 

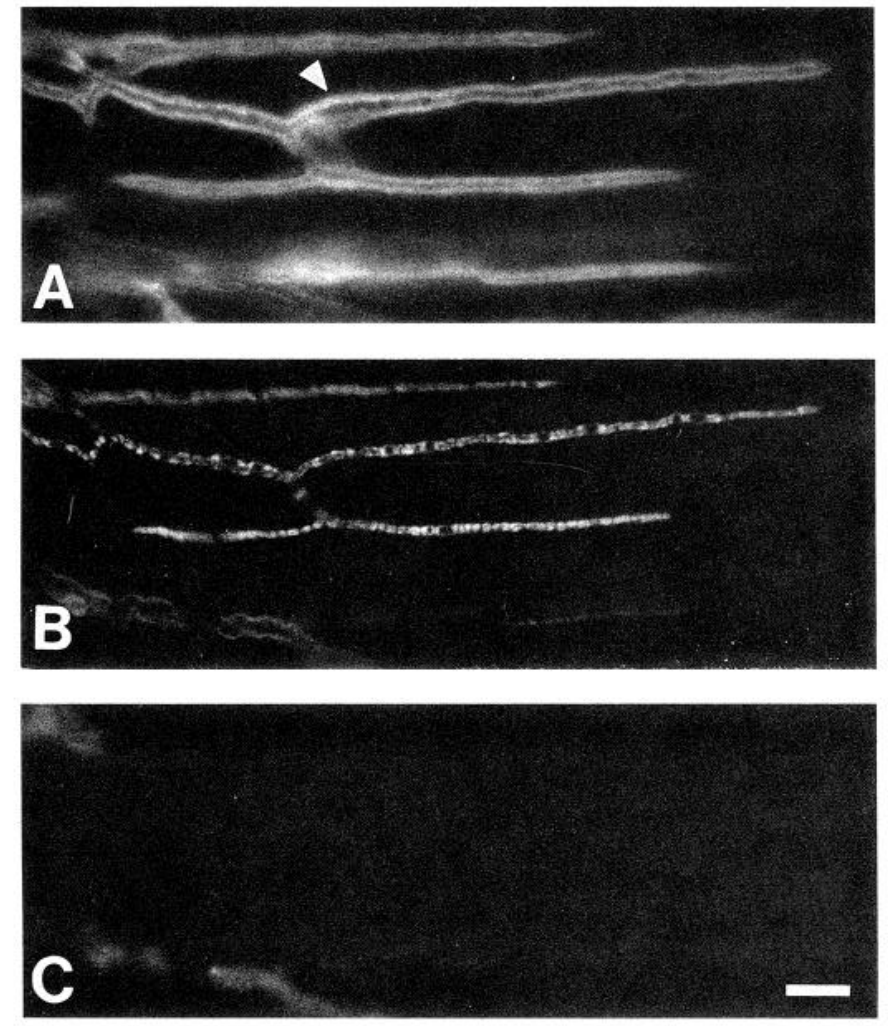

Figure 2. A nerve terminal in a normal muscle is actively stained and destained by FMI-43. A, A neuromuscular junction is marked by PNA. A terminal Schwann cell nucleus is recognized as a widening in the PNA stain (arrowhead). B, The nerve terminal, stimulated in the presence of FMI-43, is stained with bead-like fluorescence in the area delineated by the PNA stain. $C$, Further stimulation in the absence of dye caused destaining of the nerve terminal. Activity-independent staining of myelin with FM1-43 is evident in panels $B$ and $C$. Bar, $15 \mu \mathrm{m}$.

aptic site (Fig. 6C,D). These extensions of PNA stain lacked the double rail pattern normally seen at synapses (Ko, 1987), were often more weakly stained by PNA than the synaptic site, and were poorly stained by FM1-43. This pattern of PNA stain was not observed in normal preparations nor is it a response to nerve degeneration (Somasekhar and Ko, 1991). It is likely that the PNA-stained extensions are processes of terminal Schwann cells since they were continuous with PNA stain at the synaptic site and were frequently associated with nuclei (Fig. 6). One possibility is that these processes are associated with neural sprouts that overgrow the synaptic site. Such sprouts have been observed in reinnervated muscle (Letinsky et al., 1976) and basal lamina sheaths (Sanes et al., 1978) as well as in muscles whose contralateral muscles were denervated (Reichert and Rotshenker, 1979). Alternatively, the extensions of PNA stain may reflect a response of a terminal Schwann cell to the absence of muscle fibers; terminal Schwann cells of mammalian muscle elaborate fine processes after denervation (Reynolds and Woolf, 1992; Astrow et al., 1994; Son and Thompson, 1995).

Intensity of FMI-43 staining in normal and target-deprived nerve terminals

We were interested in determining whether the presynaptic function of target-deprived nerve terminals was altered by target loss when compared to the function of nerve terminals in contact with muscle fibers. To answer this question, we quantified the intensity
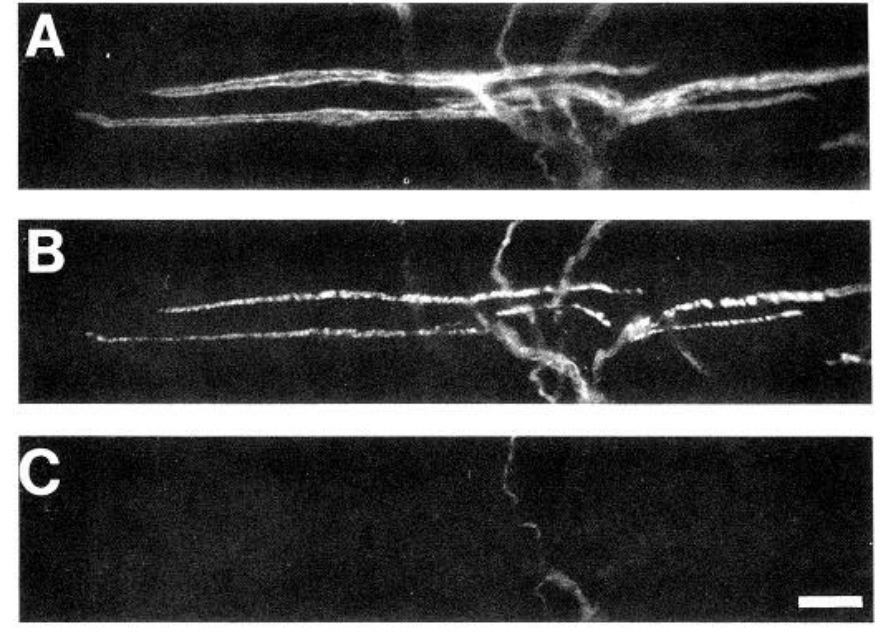

Figure 3. A nerve terminal deprived of target for 1 month is actively stained by FM1-43. A, A synaptic site in an innervated sheath preparation is stained by PNA. $B$, The nerve terminal at this synaptic site is stained by FMI-43 and $(C)$ destained upon further stimulation in the absence of the dye. Bar, $30 \mu \mathrm{m}$.
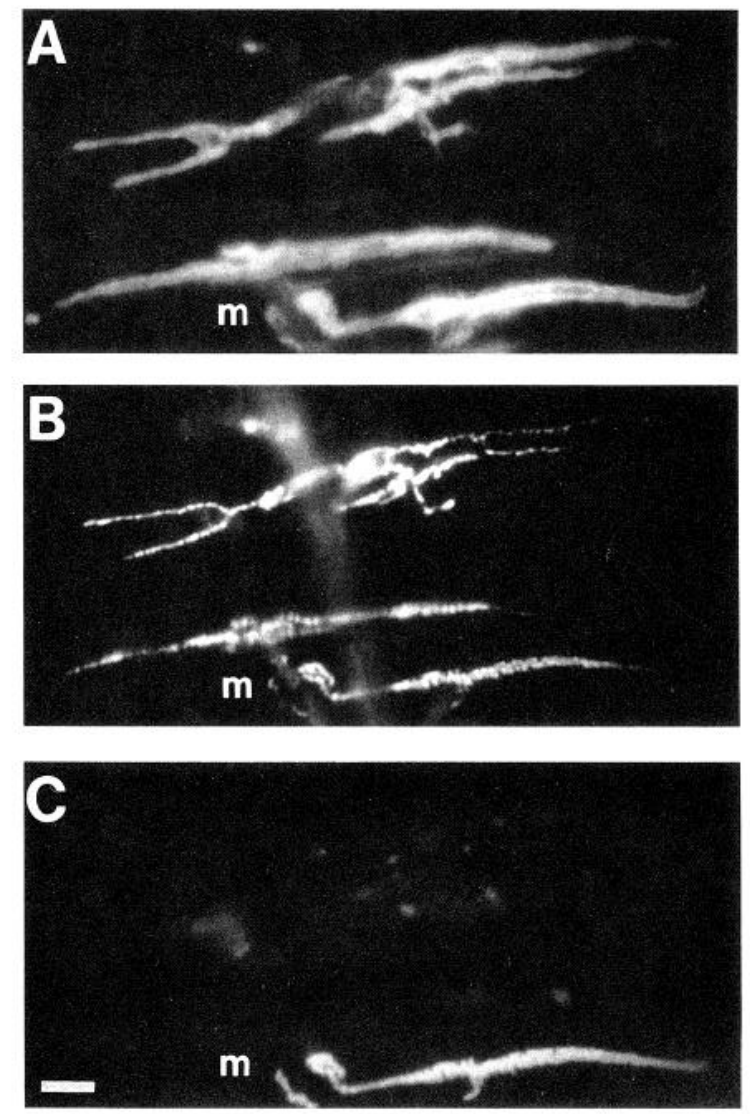

Figure 4. Target-deprived nerve terminals are stained by FMI-43 and not $\alpha$-bungarotoxin. In a 1 month innervated sheath, nerve terminals at synaptic sites marked by PNA stain $(A)$ are stained with FMI-43 (B), $C$, One of these synaptic sites, on a muscle fiber $(m)$, is stained with $\alpha$-bungarotoxin. Bar, $30 \mu \mathrm{m}$. 

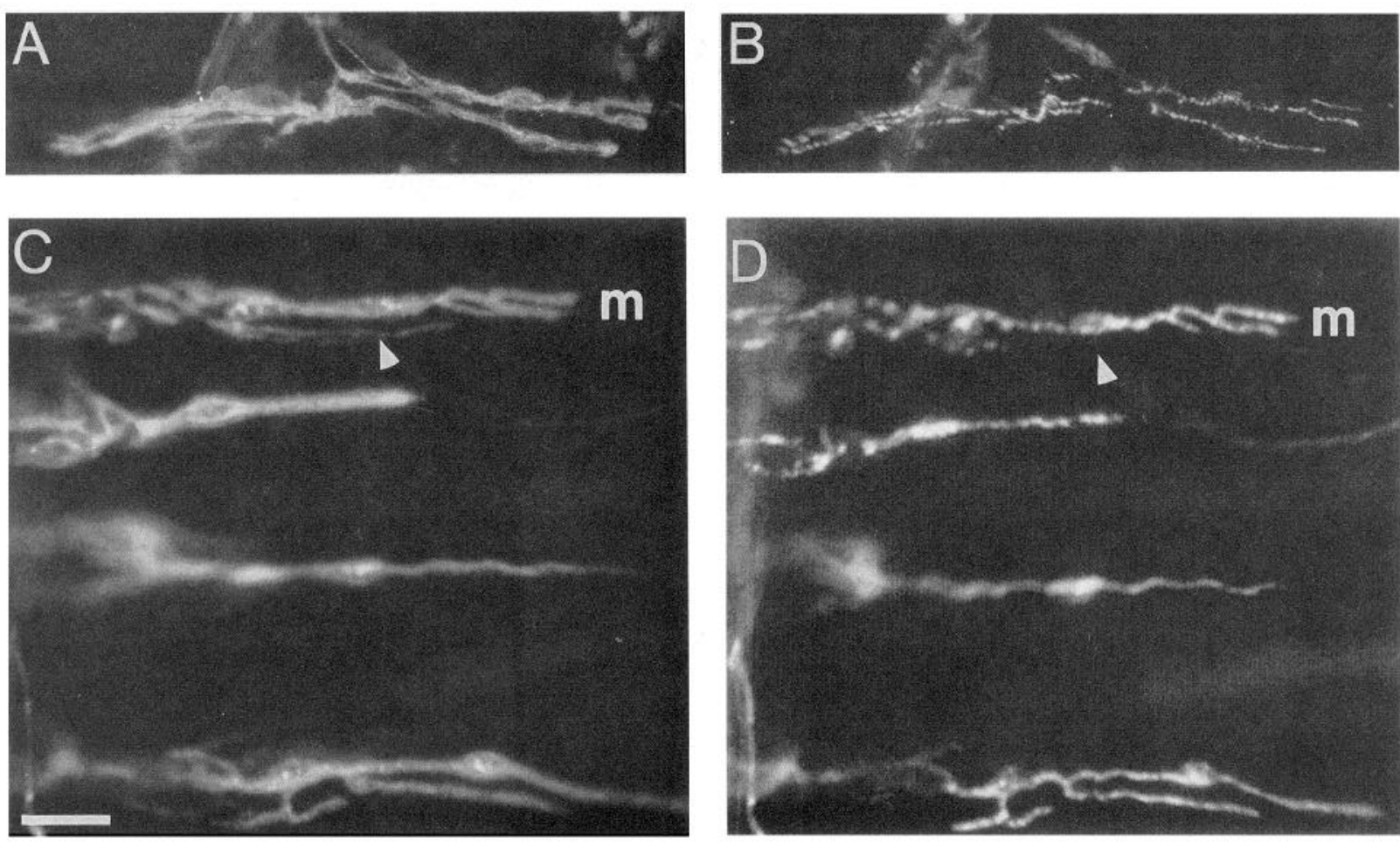

Figure 5. Functional nerve terminals are maintained at synaptic sites for several months in the absence of muscle fibers. Target-deprived synaptic sites, 3 months $(A$ and $B)$ and 5 months $(C$ and $D)$ after muscle damage, are stained by PNA $(A$ and $C$ ). The nerve terminals at these synaptic sites are functional, as demonstrated by their staining with FM1-43 $(B$ and $D)$. In a field of a 5 month innervated sheath, one nerve terminal innervates a muscle fiber that failed to degenerate $(m)$ while the remaining nerve terminals are target deprived. Some synaptic branches on the muscle fiber, marked by PNA stain, are unstained by FM1-43 (arrowheads). Bar (A and B) $36 \mu \mathrm{m}$; $(C$ and $D) 30 \mu \mathrm{m}$.

of FM1-43 stain in nerve terminals of normal muscles and innervated sheaths 1 and 5 months after muscle damage. As was evident from visual inspection, a sampling of nerve terminals revealed a range of FM1-43 staining intensities in normal and target-deprived nerve terminals (Fig. $7 A-C$ ). This is consistent with measurements of neurotransmitter release at neuromuscular junctions; release varied severalfold at frog neuromuscular junctions (Trussell and Grinnell, 1985; Yao and Weakly, 1986). When compared to normal muscle (Fig. 7A), the distributions of FM1-43 staining intensities were not significantly different in target-deprived nerve terminals from 1 and 5 month innervated sheaths (Fig. 7B,C) (Mann-Whitney test, $\alpha=0.01$ ). These results indi- cate that the level of presynaptic function in target-deprived nerve terminals is not detectably altered by muscle loss.

We also determined that this analysis of FM1-43 staining intensity was capable of detecting a reduction in presynaptic function by assaying the function of normal nerve terminals treated with $3 \mathrm{~mm}$ magnesium. At this concentration of magnesium, evoked nerve stimulation resulted in contraction of muscle fibers. Yet, the distribution of FM1-43 staining intensities was shifted dramatically reflecting a significant reduction in presynaptic function in the presence of $3 \mathrm{~mm}$ magnesium (Fig. 7D; Mann-Whitney test, $\alpha=0.01$ ). Thus, this optical assay has the sensitivity to detect a reduced level of presynaptic function.
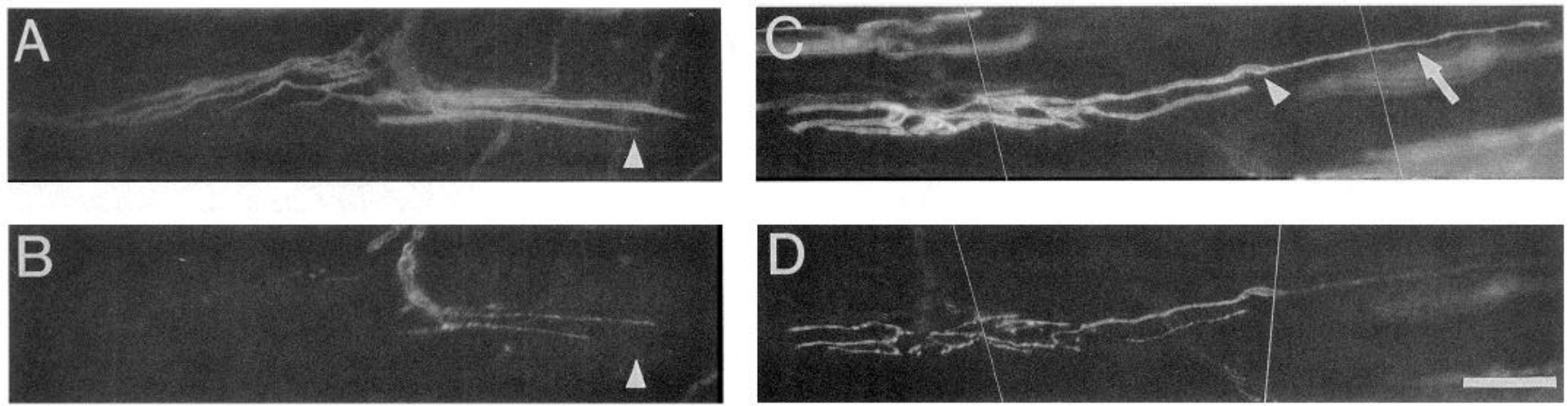

Figure 6. Altered patterns of PNA and FM1-43 stain were evident at some synaptic sites after muscle damage. $A$ and $B$, In a 1 month sheath, not all synaptic sites marked with PNA $(A)$ were stained by FM1-43 $(B)$. PNA stain is often weak at sites unstained by FM1-43. At one site, the FM1-43 and PNA stains are not coextensive (arrowheads). C, A long PNA-stained process (arrow) with an associated nucleus (arrowhead) extends from a synaptic site deprived of target for 5 months. $D$, These extensions are unstained or lightly stained by FM1-43. Bar, $50 \mu \mathrm{m}$. 
Table 1. Percentage target-deprived synaptic sites ${ }^{\prime \prime}$ positively stained by FM1-43

\begin{tabular}{llll} 
& $\begin{array}{l}\% \text { Sites } \\
\text { stained by } \\
\text { FM1-43 }\end{array}$ & \# Sites & $\begin{array}{l}\text { \# Prepara- } \\
\text { tions }\end{array}$ \\
\hline Control innervated muscle & $97 \%( \pm 3$ SD) & 183 & 4 (2 frogs) \\
1 month innervated sheath & $79 \%( \pm 8$ SD) & 186 & 3 (3 frogs) \\
5 month innervated sheath & $80 \%( \pm 7$ SD) & 357 & 3 (3 frogs)
\end{tabular}

"Synaptic sites were identified by PNA stain.
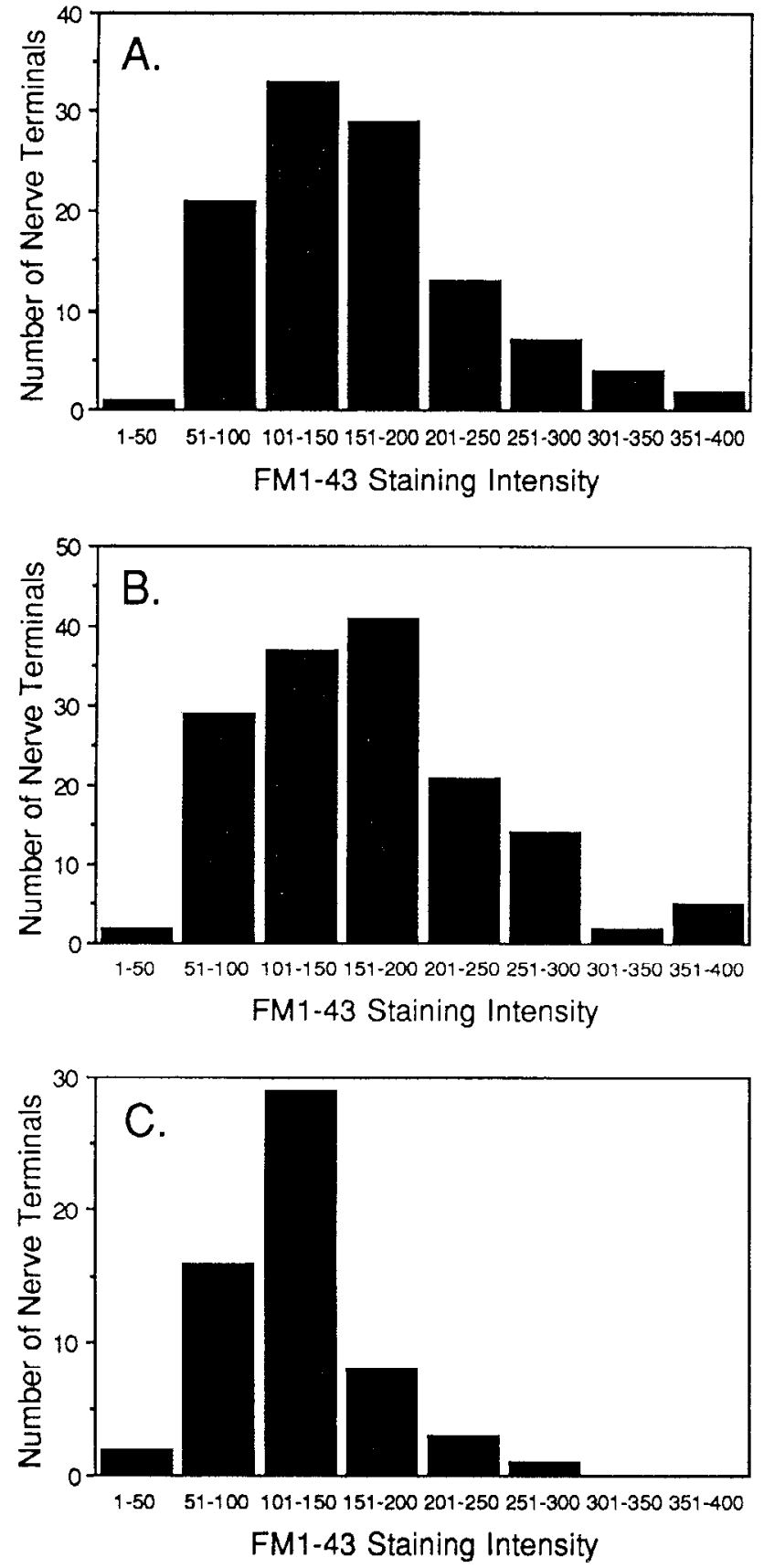

Demonstration of the maintenance of presynaptic function in target-deprived nerve terminals was also possible in innervated sheath preparations where a few muscle fibers persisted. FM143 staining of nerve terminals on surviving muscle fibers was indistinguishable from that of target-deprived terminals within the same preparation (Figs. 4, 5.) In addition, quantification revealed that the distribution of FM1-43 staining intensities of nerve terminals on surviving nuscle fibers in 1 month innervated sheaths was not statistically different when compared to 1 month target-deprived nerve terminals (Fig. 7B,E; Mann-
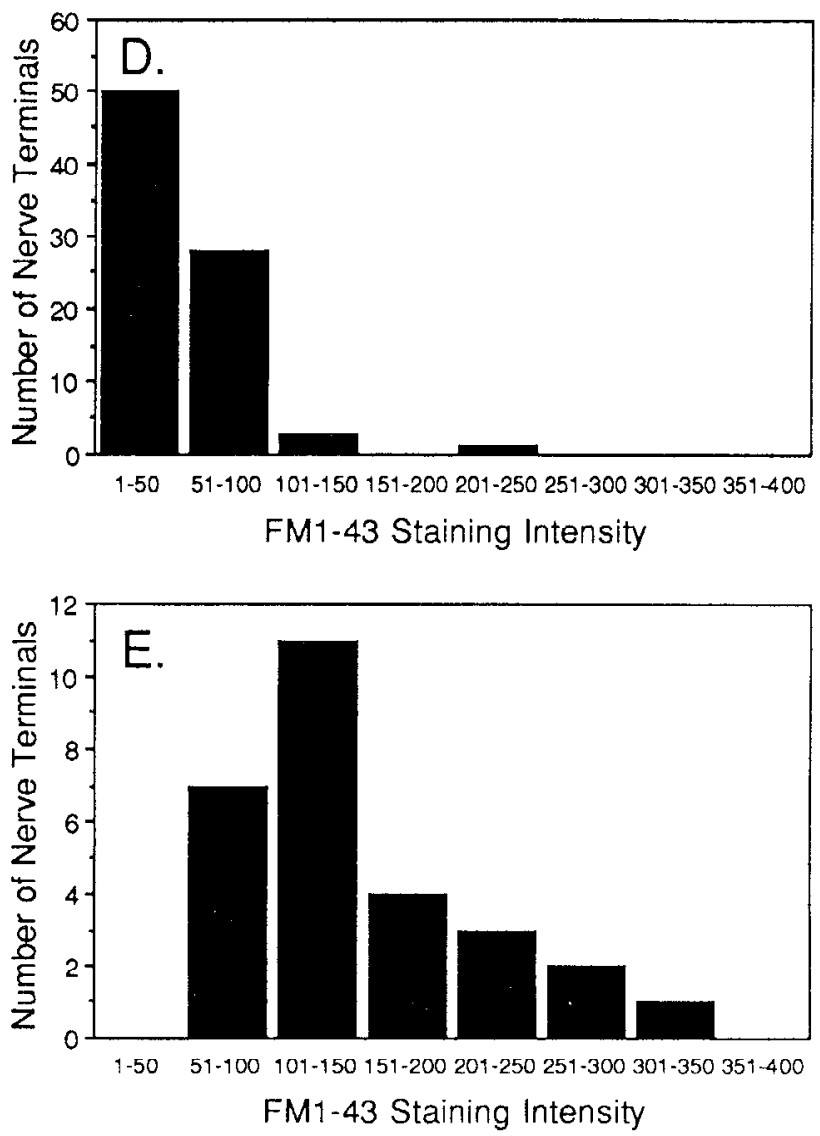

Figure 7. Presynaptic function is maintained at normal levels in target-deprived nerve terminals. The level of FM1-43 staining intensity, calculated per micron length of nerve terminal, was used as a measure of presynaptic function of nerve terminals of normal muscles and 1 and 5 months after muscle damage. The distribution of FM1-43 staining intensities is shown for $(A)$ nerve terminals in intact muscle $(n=7$ preparations), $(B)$ nerve terminals deprived of muscle fibers for 1 month $(n=5),(C)$ nerve terminals deprived of muscle fibers for 5 months $(n=3)$, $(D)$ nerve terminals in intact muscle treated with $3 \mathrm{~mm}$ magnesium $(n=4)$, and $(E)$ nerve terminals at synaptic sites on muscle fibers that persisted in innervated sheaths 1 month after muscle damage $(n=5)$. 
Whitney test, $\alpha=0.01$ ). These results suggest that the molecular apparatus required for synaptic vesicle release is maintained in a functional state in target-deprived frog nerve terminals. Therefore, the signals required for the continued synthesis and proper localization of these synaptic molecules remain intact without muscle for months.

\section{Discussion}

These studies address the role of the muscle fiber in the maintenance of presynaptic function in frog nerve terminals. Selective removal of muscle fibers produced innervated sheath preparations with original nerve terminals contacting synaptic hasal laminae. We identified nerve terminals at synaptic sites deprived of target and examined their presynaptic function with the activity-dependent dye, FM1-43. Previous studies indicated that parts of the presynaptic machinery remain functional for short times after target removal; calcium entered nerve terminals upon stimulation (Reist and Smith, 1992), and nerve terminals incorporated horseradish peroxidase immediately after muscle removal (Tal and Rotshenker, 1983) as did nerve terminals that regenerated and differentiated in the absence of target (Glicksman and Sanes, 1983). Here, we determined that 1 to 5 months after muscle fiber removal, target-deprived nerve terminals were stained by FM $1-43$ at a level indistinguishable from that of nerve terminals in normal muscle. These data demonstrate that targetdeprived nerve terminals retain their presynaptic function for long periods in the absence of muscle fibers.

We have demonstrated that a majority of target-deprived nerve terminals at PNA-stained synaptic sites are maintained in a functional state for up to 5 months in the absence of target. One question is whether PNA was a stable marker of targetdeprived synaptic sites during these experiments. If not, retraction of nerve terminals from synaptic sites accompanied by loss of PNA stain would not have been detected. Ideally, one would like to confirm that each target-deprived site remains stained by PNA. While difficult to demonstrate without repeated in vivo observation, there is evidence supporting the conclusion that no significant loss of synaptic sites occurred due to nerve terminal retraction. First, the density of synaptic sites in innervated sheaths appeared similar to that in intact muscle (Figs. 1 and 5). Large numbers of PNA-stained synaptic sites were identified in 5 month innervated sheaths, although only sitcs in myofiber-free regions were counted (Table 1 ). Note that innervated sheaths are fragile, and occasionally portions are damaged during dissection and thus unavailable for analysis. Second, we believe that PNA is a reasonable marker of synaptic sites since it remains at synaptic sites up to 5 months after denervation of muscle (Somasekhar and Ko, 1991). Although the stability of PNA at denervated sites in the absence of muscle may differ, we found that PNA continued to stain synaptic sites in 5 week denervated sheaths (Dunaevsky and Connor, unpublished observations). Third, if nerve terminal retraction was an ongoing process following muscle removal, one would expect a number of PNAstained synaptic sites to display partial FM1-43 stain, but no greater mismatch was observed at target-deprived sites than at intact neuromuscular junctions (Figs. 5, 6, Chen et al., 1991). Finally, the morphology of frog nerve terminals deprived of target for 1-5 months provides minimal cvidence of nerve terminal retraction. These terminals retained their mature differentiated structure with no evidence of degeneration (Yao, 1988b; Anglister, 1991). There was, however, an increased incidence of wrapping of nerve terminals by Schwann cell processes after several months of target deprivation (Yao, 1988b). Ensheathment of nerve terminals has been observed during synapse elimination (Korneliussen and Jansen, 1976; Riley, 1981) and target deprivation (Jirmanova, 1975). Whether this ensheathment of nerve terminals is indicative of retraction is unknown. What is clear is that the late onset of nerve terminal ensheathment ( 3 months, Yao, 1988b) ensures that a synaptic site vacated by possible nerve retraction would still be marked by PNA stain at the latest time point examined in these experiments ( 5 months). Although we cannot climinate the possibility that nerve terminal retraction and loss of identified synaptic sites occurs in the $a b$ sence of muscle, we conclude that its magnitude at target-deprived nerve terminals of the frog is minimal in the time frame examined here.

There is evidence that muscle does contribute to the stability of nerve terminals; mammalian nerve terminals and regenerating frog nerve terminals both require muscle for maintenance (Sanes et al., 1978; Yao, 1988a; Rich and Lichtman, 1989). In striking contrast to these data is the result presented here demonstrating that presynaptic function is maintained in target-deprived nerve terminals for months. What do these findings suggest about the contribution of muscle to the maintenance of presynaptic function in frog nerve terminals? One possibility is that a motor unit need only contact a few muscle fibers to stabilize all synaptic sites; a few fibers might provide sufficient signal to maintain the presynaptic activity of an entire motor unit. However, we found that nerve terminals were stained by FM1-43 in 6 innervated sheaths that were completely devoid of muscle fibers, indicating that nerve terminals of motor units totally deprived of target retain their presynaptic function.

Another explanation for the observed maintenance of presynaptic function in target-deprived nerve terminals is that a stability signal, lost after muscle removal, has a long-lasting effect on nerve terminals. Alternatively, a stability signal produced by a muscle fiber may be deposited in the synaptic basal lamina and persist after muscle fiber removal. Agrin, the matrix molecule thought to be responsible for the postsynaptic aggregation of AChRs and acetylcholinesterase, is bound to the synaptic basal lamina and remains functional in the absence of muscle (McMahan and Slater, 1984; Anglister and McMahan, 1985). Such a role for molecules bound to the basal lamina in nerve terminal stability is supported by evidence that basal lamina removal by proteolytic enzymes caused the disruption of active zones (Nystrom and Ko, 1988). One hypothesis is that such a stability signal may contribute to an adhesive bridge binding presynaptic and postsynaptic elements (Balice-Gordon and Lichtman, 1990). The rapid retraction of mammalian nerve terminals after target loss is thought to be due to disruption of such adhesive connections (Rich and Lichtman, 1989). In the frog, niuscle fiber removal may not be as disruptive to these proposed connections, although we and others found no evidence of remnants of postsynaptic membrane, assayed by the presence of AChRs, at target-deprived synaptic sites (Fig. 4, Rieger et al., 1988). In addition, the loss of a stability factor in the frog may be slowed when compared to mammals due to temperature differences. Regardless of the nature of the stability signal, it is clear that it is able to maintain only nerve terminals that have experienced contact with muscle fibers; nerve terminals that reinnervate basal lamina sheath preparations form differentiated nerve terminals that are not stabilized (Sanes et al., 1978; Glicksman and Sanes, 1983; Yao, 1988a). The remarkable stability of functional frog nerve terminals in the absence of target provides an interesting system in which to inves- 
tigate further the nature of molecules important in the maintenance of synaptic connections.

\section{References}

Anglister L (1991) Acetylcholinesterase from the motor nerve terminal accumulates on the synaptic basal lamina of the myofiber. $\mathbf{J}$ Cell Biol 115:755-764.

Anglister LA, McMahan UJ (1985) Basal lamina directs acetylcholinesterase accumulation at synaptic sites in regenerating muscle. J Cell Biol 101:735-743.

Astrow S, Son Y-J, Thompson W (1994) Differential neural regulation of a neuromuscular junction-associated antigen in muscle fibers and Schwann cells. J Neurobiol 25:937-952.

Balice-Gordon RJ, Lichtman JW (1990) In vivo visualization of the growth of pre- and postsynaptic elements of neuromuscular junctions in the mouse. J Neurosci 10:894-908.

Balice-Gordon RJ, Lichtman JW (1994) Long-term synapse loss induced by focal blockade of postsynaptic receptors. Nature 372:519 524.

Bennett MK, Calakos N, Scheller RH (1992) Syntaxin: a synaptic protein implicated in docking of synaptic vesicles at presynaptic active zones. Science 257:255-259.

Betz WJ, Mao F, Bewick GS (1992) Activity-dependent fluorescent staining and destaining of living vertebrate motor nerve terminals. $J$ Neurosci 12:363-375.

Bowe MA, Fallon JR (1995) The role of agrin in synapse formation. Annu Rev Neurosci 18:443-462.

Chen L, Ko C-P (1994) Extension of synaptic extracellular matrix during nerve terminal sprouting in living frog neuromuscular junctions. J Neurosci 14:796-808.

Chen L, Folsom DB, Ko C-P (1991) The remodeling of synaptic extracellular matrix and its dynamic relationship with nerve terminals at living frog neuromuscular junctions. J Neurosci 11:2920 2930.

Cohen MW, Jones OT, Angelides KJ (1991) Distribution of $\mathrm{Ca}^{2+}$ channels on frog motor nerve terminals revealed by $\omega$-conotoxin. $J$ Neurosci 11:1032-1039.

Connor EA, McMahan UJ (1987) Cell accumulation in the junctional region of denervated muscle. J Cell Biol 104:109-120.

Connor EA, Qin K, Yankelev H, DeStefano D (1994) Synaptic activity and connective tissue remodeling in denervated frog muscle. J Cell Biol 127:1435-1445.

Connor EA, Smith MA (1994) Retrograde signaling in the formation and maintenance of the neuromuscular junction. J Neurobiol 25:722739.

Falls DL, Harris DA, Johnson FA, Morgan MM, Corfas G, Fischbach GD (1990) $M, 42,000$ ARIA: a protein that may regulate the accumulation of acctylcholine receptors at developing chick neuromuscular junctions. Cold Springs Harb Symp Quant Biol 55:397-406.

Falls DL, Rosen KM, Corfas G, Lane WS, Fischbach GD (1993) ARIA, a protein that stimulates acetylcholine receptor synthesis, is a member of the neu ligand family. Cell 72:801-815.

Glicksman M,Sanes JR (1983) Differentiation of motor nerve terminals formed in the absence of muscle fibers. J Neurocytol 12:661-671.

Herrera AA, Werle MJ (1990) Mechanisms of elimination, remodeling, and competition at frog neuromuscular junctions. I Neurobiol 21:7198.

Herrera AA, Banner LR, Nagaya N (1990) Repeated, in vivo observation of frog neuromuscular junctions: remodeling involves concurrent growth and retraction. J Neurocytol 19:85-99.

Jirmanova I (1975) Ultrastructure of motor end-plates during pharmacologically-induced degeneration and subsequent regeneration of skeletal muscle. J Neurocytol 4:141-155.

Ko C-P (1987) A lectin, peanut agglutinin, as a probe for the extracellular matrix in living neuromuscular junctions. J Neurocytol 16: 567-576.

Korneliussen H. Jansen J (1976) Morphological aspects of the elimination of polyneuronal innervation of skeletal muscle fibres in newborn rats. J Neurocytol 5:591-604.

Kuffler SW, Yoshikami (1975) The distribution of acetylcholine sensitivity at the post-synaptic membrane of vertebrate skeletal twitch muscles: Iontophoretic mapping in the micron range. J Physiol (Lond) 244:703-730.

Letinsky MK, Fischbach GD, McMahan UJ (1976) Precision of reinnervation of original postsynaptic sites in frog muscle after a nerve crush. J Neurocytol 5:691-718.

Lichtman JW, Magrassi L, Purves D (1987) Visualization of neuromuscular junctions over periods of several months in living mice. J Neurosci 7:1215-1222.

McMahan UJ, Slater CR (1984) The influence of basal lamina on the accumulation of acetylcholine receptors at synaptic sites in regenerating muscle. J Cell Biol 98:1453-1473.

Noakes PG, Gautam M, Mudd J, Sanes JR, Merlie JP (1995) Aberrant differentiation of neuromuscular junctions in mice lacking s-laminin/ laminin $\beta 2$. Nature 374:258-262.

Nystrom RR, Ko CP (1988) Disruption of active zones in frog neuromuscular junctions following treatment with proteolytic enzymes. J Neurocytol 17:63-71.

Ramaswami M, Krishnan KS, Kelly RB (1994) Intermediates in synaptic vesicle recycling revealed by optical imaging of Drosophila neuromuscular junctions. Neuron 13:363-375.

Reichert F, Rotshenker S (1979) Motor axon terminal sprouting in intact muscles. Brain Res 170:187-189.

Reist NE, Smith SJ (1992) Neurally-evoked calcium transients in terminal Schwann cells at the neuromuscular junction. Proc Natl Acad Sci USA 89:7625-7629.

Reynolds ML, Woolf CJ (1992) Terminal Schwann cells elaborate extensive processes tollowing denervation of the motor endplate. J Neurocytol 21:50-66.

Rich M, Lichtman JW (1989) Motor nerve terminal loss from degenerating muscle fibers. Neuron 3:677-688

Rieger F, Nicolet M, Pincon-Raymond M, Crossin KL, Grumet M, Edelman GM (1988) Distribution and role in regeneration of N-CAM in the basal lamina and Schwann cells. J Cell Biol 107:707-719.

Riley D (1981) Ultrastructural evidence for axon retraction during the spontaneous elimination of polyneuronal innervation of the rat soleus muscle. J Neurocytol 10:425-440.

Robitaille R, Adler EM, Charlton MP (1990) Strategic localization of calcium channels at neurotransmitter relcase sitcs of the frog neuromuscular synapses. Neuron 5:773-779.

Robitaille R, Garcia ML, Kaczorowski GJ, Charlton MP (1993) Functional colocalization of calcium and calcium-gated potassium channels in control of transmitter release. Neuron 11:645-655.

Ryan TA, Reuter H, Wendland B, Schweizer FE, Tsien RW, Smith SJ (1993) The kinetics of synaptic vesicle recycling measured at single presynaptic boutons. Neuron 11:713-724.

Sanes JR, Marshall LM, McMahan UJ (1978) Reinnervation of muscle fiber basal lamina after removal of myofibers. J Cell Biol 78:176-198.

Somasekhar T, Ko C-P (1991) Effects of denervation on the distribution of peanut agglutinin hinding molecules in frog muscles. I Neurocytol 20:65-77.

Son Y-J, Thompson W (1995) Nerve sprouting in muscle is induced and guided by processes extended by Schwann cells. Neuron 14:133141.

Tal M, Rotshenker S (1983) Recycling of synaptic vesicles in motor nerve endings separated from their target muscle fibers. Brain Res 270:131-133.

Trussell LO, Grinnell AD (1985) The regulation of synaptic strength within motor units of the frog cutaneous pectoris muscle. J Neurosci $1: 243-254$.

Ushkaryov YA, Petrenko AG, Geppert M, Südhoff TC (1992) Neurexins: synaptic cell surface proteins related to the $\alpha$-latrotoxin receptor and laminin. Science 257:50-56.

Wernig A, Herrera AA (1986) Sprouting and remodelling at the neuromuscular junction. Prog Neurobiol 27:251-291.

Yao YM (1988a) Factors that influence the maintenance of normal and regenerating axon terminals. In: The current status of peripheral nerve regeneration, pp 235-246. New York: Liss.

Yao YM (1988b) Maintenance of axon terminals at synaptic sites in the absence of muscle fibers. In: Current issues in neural regeneration research, pp 167-178. New York: Liss.

Yao Y, Weakly N (1986) Differences in transmitter release and number of nerve terminals per motoneuron between two frog muscles. I Neu rosci $6: 498-506$. 